\title{
Multi-Objective Optimal Placement of Recloser and Sectionalizer in Electricity Distribution Feeders
}

\author{
Arash Zeinalzadeh \\ North Khorasan Electric Distribution \\ Company \\ Bojnourd, Iran \\ zeynalzadeharash@yahoo.com
}

\author{
Abouzar Estebsari \\ Department of Energy \\ Politecnico di Torino \\ Turin, Italy \\ abouzar.estebsari@polito.it
}

\author{
Alireza Bahmanyar \\ Department of Electrical Engineering \\ Iran University of Science and \\ Technology \\ Tehran, Iran \\ bahmanyar@iust.ac.ir
}

\begin{abstract}
Electricity distribution feeders, due to their geographical dispersion, are subjected to faults caused by adverse weather, vegetation growth, etc., resulting in long outages for customers. Overhead switching devices (i.e. reclosers, sectionalizers, disconnectors and etc.) are known as the most practical solutions to limit the outage area, and consequently increase the distribution system reliability. This paper presents a Multi-Objective Particle Swarm Optimization (MOPSO) algorithm to minimize customers' outage, maintenance and investment costs, and increase system reliability. The algorithm determines the number and optimal locations of reclosers and sectionalizers to fulfill the objectives. The obtained results on the standard 85-node distribution feeder validate the effectiveness of the proposed algorithm.
\end{abstract}

Keywords- Distribution system, MOPSO, Optimization, Recloser, Sectionalizer

\section{INTRODUCTION}

Ensuring system reliability is known as one of the main challenges distribution companies face. Wide geographical dispersion of electricity distribution networks in urban and rural areas subjects them to different kinds of short circuit faults caused by a variety of causes, such as adverse weather, vegetation growth, bird contact and etc. As the final stage of the electrical energy supply chain, any sustained fault on distribution systems results in customers outage and affects the system reliability. Distribution companies take much effort to limit the outage area and keep the lights of the nonaffected parts of the network on. The use of overhead switching devices such as reclosers and sectionalizers is known as the most practical solution in this regard.

Most of the faults on overhead electricity distribution networks are transient in nature, commonly caused by lightning and temporary contacts. Transient faults can be successfully cleared by immediate tripping and reenergization of the line. On overhead distribution feeders, autoreclosers do this tripping followed by re-energization process.

A recloser can work in coordination with a number of downstream sectionalizers. For temporarily faults, sectionalizers which count the interruptions made by the upstream recloser, after a number of reclosing shots, isolate the faulty downstream network during the dead time of the recloser to limit the outage area. Optimal placement of reclosers and sectionalizers on a distribution feeder can limit the outage area, improve system reliability, and decrease the required investment and maintenance costs.
Different optimization techniques are employed for optimal placement of switching devices in distribution systems. These include genetic algorithm [1], simulated annealing [2], binary programming [3], Bellmann's optimality principle [4], ant colony algorithm [5], immune algorithm [6], and particle swarm optimization [7].

In [8] authors consider the effect of using reclosers and remote disconnectors on the system reliability. The authors of [9] propose a heuristic combinatory search algorithm to determine the optimal level of investments in medium voltage distribution network automation. In [10] a methodology is implemented for allocation of remotely controlled switching devices in electric distribution systems based on the analytic hierarchical process method. The method proposed in [11] proposes a modified shuffled frog leaping algorithm to derive the optimal placement of manual and automatic switches. The authors of [12] propose a new mathematical model to calculate the restorative energy and use the genetic algorithm to optimize the location of the switches. The method proposed in [13] uses the Mixed-integer linear programming for sectionalizing switch placement.

The authors in [14] propose a multi-objective optimization approach for switch placement based on the particle swarm optimization method. In $[15,16]$ authors consider remotely controlled switches and propose different algorithms for their placement. The method proposed in [17] determines the optimal composition and placement of automatic switches.

This paper presents a Multi-Objective Particle Swarm Optimization (MOPSO) algorithm to minimize customers outage, maintenance and investment costs and increase system reliability as the objective functions by optimal placement of reclosers and sectionalizers in radial distribution feeders. The method performance is evaluated on the standard 85-node distribution feeder. The results validate the effectiveness of the proposed algorithm to fulfil the objectives.

The contents of this paper is structured as follows: in Section II, the proposed method based on multi-objective optimization is described. Section III demonstrate the performance of this solution by some simulation results on 85 node distribution network. The paper is concluded with some remarks in Section IV.

\section{THE PROPOSED METHOD}

The overall structure of the proposed method is consisted of three parts, an optimizer, a fault case simulator and a fuzzy compromiser. At the first step the method receives system data (network configuration, loads and etc.) and parameters (failure rates, energy cost and etc.). It then considers random numbers 
and locations of overhead switches as a set of particles. The switches random locations are considered at the beginning of sections. At the next step the method runs the fault case simulator for particles and calculates the objective function for each. Then, the optimizer updates the particles positions and velocities (number and locations of switches). The fault case simulator calculates the objective functions for updated particles and the process repeats until the calculated objective function converges. Finally, a fuzzy compromiser selects the final solution. The method building blocks are described in the following subsections. It should be noted that a recloser (with three shots) cannot be coordinated with more than three downstream sectionalizers.

\section{A. Objective function}

Customers outage duration and cost, network topology, loads variations, network components failure rate, the required time to clear the fault or to isolate the faulted part of the network, and reliability improvements are among the factors that must be considered for definition of the objective function.

To consider the cost of customers outage, the following function proposed in [18] is taken as the first objective:

$$
F_{1}=E_{C O S T}=\sum_{j=1}^{N C} \sum_{k=1}^{N L L} L_{k j} \cdot C_{k j}\left(r_{j}\right) \cdot \lambda_{j}
$$

where:

$N I L=$ total number of load points isolated due to event $j$;

$N C=$ number of outage events (Contingencies);

$L_{k j}=$ load outage at point $k$ for event $j$;

$r_{j}=$ outage duration for event $j$;

$\lambda_{j}=$ average failure rate of event $\mathrm{j}$ for study duration;

$C_{j k}\left(r_{j}\right)=$ outage cost $(\$ / \mathrm{KW})$ of load point $k$ for event $j$

with an outage duration of $r_{j}$.

The second objective function considers the improvement of reliability indices including system average interruption frequency index (SAIFI) and system average interruption duration index (SAIDI):

$$
F_{2}=w_{1} \cdot S A I F I+w_{2} \cdot S A I D I
$$
0.5 .

where $w_{l}$ and $w_{2}$ are weighting coefficients both equal to

The result of the optimal placement problem must be economically viable for distribution companies, considering the price of switching equipment, their installation cost and the large scale of distribution networks. Therefore, the cost of the switching equipment is considered in the following function, as the third objective:

$$
F_{3}=n \cdot C_{S E C}+m \cdot C_{R E C}
$$

where $n$ and $m$ are the number of sectionalizers and reclosers, respectively and $C_{S E C}$ and $C_{R E C}$ denote their prices.

\section{B. Optimization algorithm}

Particle swarm optimization (PSO) is a population based stochastic optimization algorithm inspired by co-operative intelligence of the swarm such as bird flocking. The algorithm uses a population of individuals to probe the best position in the search space. It is comprised of a set of particles moving around the search space. The position of each particle is modified based on its own and its neighbors experiences.

For simultaneous optimization of multiple objective functions, the multi-objective PSO is proposed [19]. This algorithm considers a vector $x^{*}$ as Pareto optimal, if any other feasible vector which would decrease some objectives, causes an increase in other objectives. The set of optimal solutions of the MOPSO is known as the Pareto front, including a set of non-dominated solutions.

If $x_{1}$ is not less optimal than $x_{2}$, for any of the objective functions and it is more optimal for at least one function, $x_{I}$ is said to dominate $x_{2}$.

The MOPSO algorithm follows the basic relationship of the single-objective version of the particle pool optimization. The steps of the algorithm are as follows:

1. The initial positions and velocities of all particles are generated randomly. In the optimization case considered, the location of switches are generated in form of vectors as algorithm particles.

2. Objective functions calculation.

3. Identification of non-dominated solutions.

4. The non-dominated solutions are selected and stored in a repository.

5. Update velocity and position of each particle. Considering that the algorithm has a set of optimal solutions, a leader should be chosen for each particle. The best position of each particle should be updated in this step. Therefore, for each particle, by comparing the new position to the best global position, the dominant one will be selected as the new position. If none of the particles dominates, a random one will be selected.

6. Update the best position of each particle by comparing the new position to the best global position and selecting the dominant one. If none of them dominates, a random one will be selected.

7. After all the particles are updated, store the current population non-dominated members in the repository.

8. Remove the dominated solution of the repository and keep the non-dominated ones.

9. If the repository members exceeds a predefined maximum, remove the extra members.

10. If the algorithm converges, the optimization process is over. Otherwise, go to step 5.

11. Select the non-dominated population as the Pareto front.

The resulted Pareto front is a set of optimal solutions. The final solution can be selected based on distribution system engineers expertise and their requirements. However, due to the inherent uncertainty in human decision-making, the fuzzy compromise technique is proposed for the selection of the final solution.

\section{Fuzzy compromise}

In this method, for objective function $i$ for the solution $k$ of the Pareto front, a $\mu_{i}^{k}$ value is calculated: 


$$
\mu_{i}^{k}=\left\{\begin{array}{cc}
1 & f_{i} \leq f_{i}^{\min } \\
\frac{f_{i}^{\max }-f_{i}}{f_{i}^{\max }-f_{i}^{\min }} & f_{i}^{\min } \leq f_{i} \leq f_{i}^{\max } \\
0 & f_{i} \geq f_{i}^{\max }
\end{array}\right.
$$

where $f_{i}^{\text {max }}$ and $f_{i}^{\text {min }}$ are the min and max of the objective function $i$ of all solutions in the Pareto front.

The fuzzy membership function for solution $k$ can be calculated as follows:

$$
\mu^{k}=\max _{k=1}^{N}\left(\min \left(\sum_{i=1}^{m} \mu_{i}^{k}\right)\right)
$$

The solution with the maximum $\mu^{k}$ will be selected as the best solution.

\section{Fault case simulation}

The method has to consider different possible fault locations for the optimal placement problem. The steps of fault case simulation for calculation of the objective function are presented in Fig. 1. In this flowchart, the time for fault clearance and reconnection of the isolated section and the time from the isolation of the affected section by the sectionalizer are denoted by $t_{r e p}$ and $t_{\text {sec }}$ respectively.

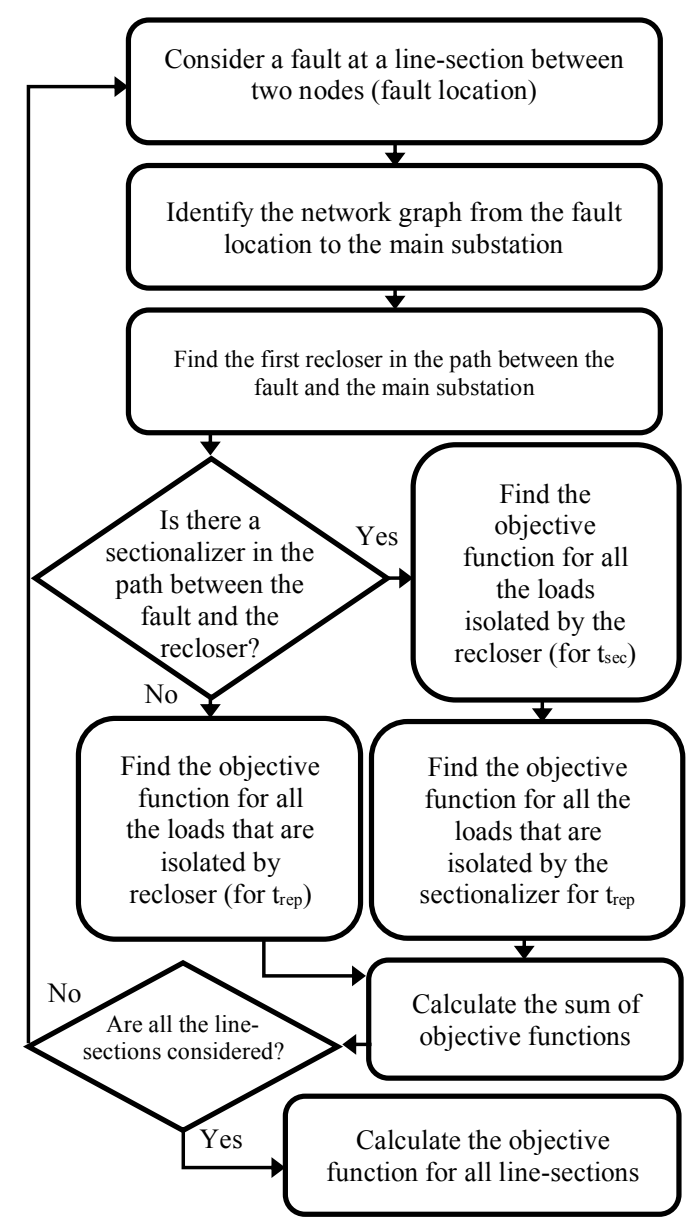

Fig. 1. The steps of fault case simulation for calculation of the objective function

\section{SIMULATION TEST RESULTS}

In this section, the ability of the proposed method to find the optimal locations and number of reclosers and sectionalizers is evaluated. The tests are done on the standard 85-node distribution feeder [20].

Table I presents the considered load types and Table II shows the parameters considered for this study. Table III presents the identified optimal number and locations of the reclosers and sectionalizers. The results are also shown in Fig. 2. The results indicate that with optimal placement of reclosers and sectionalizers, compared to the case with no overhead switches, an expected net revenue of $46168 \$$ per year can be made. The results matches the engineering judgments.

TABLE I. LOAD TYPES CONSIDERED FOR EACH NODE

\begin{tabular}{|l|l|}
\hline \multicolumn{1}{|c|}{ Load type } & \multicolumn{1}{c|}{ Node } \\
\hline Small-scale industrial load & $14,15,16,17,18,19,20$ \\
\hline Commercial loads & $59,60,61,71,72,82,83,84,85$ \\
\hline Domestic loads & Other nodes \\
\hline
\end{tabular}

TABLE II. CONSIDERED PARAMETERS

\begin{tabular}{|l|l|}
\hline \multicolumn{1}{|c|}{ Parameter } & \multicolumn{1}{c|}{ Considered value } \\
\hline$\lambda$ & $0.065(\mathrm{f} / \mathrm{yr} / \mathrm{km})[2]$ \\
\hline trep & 1 hour \\
\hline tsec & 9 second \\
\hline Recloser price & $11800 \$$ \\
\hline Sectionalizer price & $4700 \$$ \\
\hline Switches life time & 20 years \\
\hline Outage cost for domestic loads & $0.162 \$ \mathrm{~kW} \mathrm{[1]}$ \\
\hline Outage cost for industrial loads & $3.32 \$ \mathrm{~kW} \mathrm{[1]}$ \\
\hline $\begin{array}{l}\text { Outage cost for commercial } \\
\text { loads }\end{array}$ & $9.97 \$ \mathrm{~kW} \mathrm{[1]}$ \\
\hline Study period & 1 year \\
\hline Time horizon & 10 year \\
\hline
\end{tabular}

TABLE III. THE OPTIMAL NUMBER AND LOCATIONS OF OVERHEAD SWITCHES

\begin{tabular}{|l|l|}
\hline Number of reclosers & 3 \\
\hline $\begin{array}{l}\text { Reclosers optimal locations } \\
\text { (line-sections) }\end{array}$ & $25-8,57-9,4-5$ \\
\hline Number of sectionalizers & 8 \\
\hline $\begin{array}{l}\text { Sectionalizers optimal } \\
\text { locations (line-sections) }\end{array}$ & $\begin{array}{l}5-18,9-10,12-80,60-63-64- \\
67,29-30,34-44,35-48\end{array}$ \\
\hline $\begin{array}{l}\text { Ecost before installation } \\
\text { (\$/yr) }\end{array}$ & 238726 \\
\hline $\begin{array}{l}\text { Ecost after installation } \\
\text { (\$/yr) }\end{array}$ & 42518 \\
\hline $\begin{array}{l}\text { Total price of overhead } \\
\text { switches }\end{array}$ & 3650 \\
\hline Total cost & 35223 \\
\hline Expected revenue (\$/yr) & 46168 \\
\hline F2 before installation & 6.8 \\
\hline F2 after installation & 2.2 \\
\hline
\end{tabular}




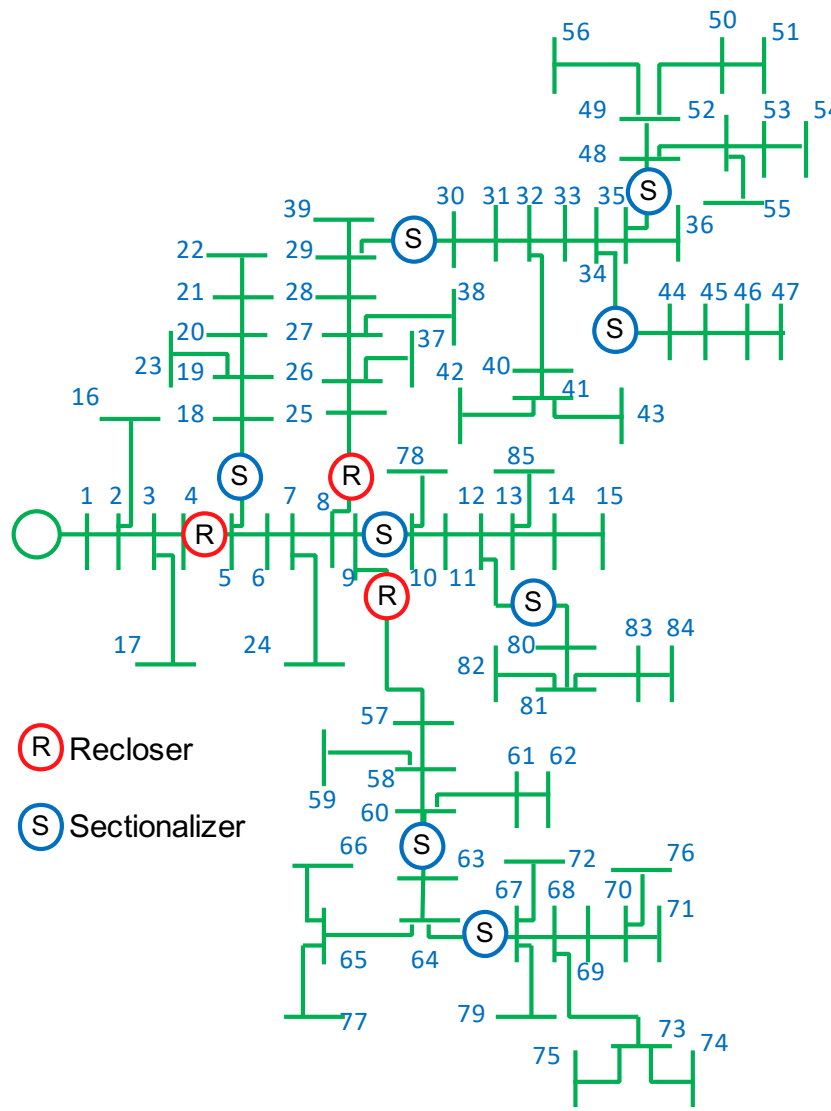

Fig. 2. Identified optimal number and locations of the reclosers and sectionalizers for 85 -node test feeder

\section{CONCLUSIONS}

An MOPSO algorithm is presented for optimal placement of reclosers and sectionalizers in radial distribution systems. As its objectives, the algorithm aims to increase system reliability, and to minimize customers outage time, maintenance costs and investment costs.

The obtained results on the standard IEEE 85-node distribution feeder validate the effectiveness of the proposed algorithm. The obtained locations and numbers of the switching devices match the engineering judgments. The results indicate that with optimal placement of reclosers and sectionalizers, compared to the case with no overhead switches, the system reliability increases considerably, and an expected net revenue of 46168 \$ per year can be made.

\section{REFERENCES}

[1] G. Levitin, M. Tov, D. Elmakis,"Optimal Sectionalizer Allocation in Electric Distribution Systems by Genetic Algoritm", Electric Power System Research, Vol. 31, pp. 97-102, nov.1994.

[2] R. Billinton, S. Jonnavithula,"Optimal Switching Device Placement in Radial Distribution System", IEEE Transactions Power Delivery, Vol. 13, No. 1, pp. 218-224, Jan. 1998.
[3] F. Soudi, K. Tomsovic,"Optimized Distribution Protection Using Binary Programming", IEEE Transactions Power Delivery, Vol. 13, No. 1, pp. 218-224, Jan. 1998.

[4] G. Celli, F. Pilo,"Optimal Sectionalizing Switches Allocation in Distribution Network", IEEE Transactions Power Delivery, Vol. 13, No. 3, pp. 1167-1172, July 1999.

[5] J. H. Teng,"A Novel ACS-Based Optimal Switch Relocation Method", IEEE Transactions Power Delivery, Vol. 13, No. 1, Feb. 2003.

[6] C. S. Chen, C. H. Lin,"Optimal Placement of Line Switches for Distribution Automation Systems using Immune Algorithm", IEEE Transactions Power Delivery, Vol. 13, No. 3, Aug. 2006.

[7] Moradi, M. Fotuhi,"Optimal Switch Placement in Distribution Systems Using Trinary Particle Swarm Optimization Algorithm", IEEE Transactions Power Delivery, Vol. 13, No. 1, Jan. 2008.

[8] J. Haakana et al. "Comparison of Reliability Indices from the Perspective of Network Automation Devices", IEEE Transactions Power Delivery, Vol. 25, No. 3, pp. 1547-1555, 2010.

[9] D. S. Popovic, L. R. Glamocic, M. D. Nimrihter, "The optimal automation level of medium voltage distribution networks", Electrical Power and Energy Systems, Vol. 33, pp. 430-438, 2011.

[10] D. P. Bernardon et al. "AHP Decision-Making Algorithm to Allocate Remotely Controlled Switches in Distribution Networks", IEEE Transactions Power Delivery, Vol. 26, pp. 1884-1892, 2011.

[11] Goroohi Sardou et al. "Modified shuffled frog leaping algorithm for optimal switch placement in distribution automation system using a multi-objective fuzzy approach", IET Generation, Transmission \& Distribution, Vol. 6, pp. 493-502, 2012.

[12] H. Dezaki et al. "Optimized Switch Allocation to Improve the Restoration Energy in Distribution Systems", Journal of Elec. Engineering, Vol. 63, pp. 47-52, 2012.

[13] A. Abiri-Jahromi, M. Fotuhi-Firuzabad, M. Parvania, and M. Mosleh, "Optimized sectionalizing switch placement strategy in distribution systems," IEEE Transactions on Power Delivery, vol. 27, pp. 362-370, 2012 .

[14] J. R. Bezerra, G. C. Barroso, R. P. S. Leão, and R. F. Sampaio, "Multiobjective optimization algorithm for switch placement in radial power distribution networks," IEEE Transactions on Power Delivery, vol. 30, pp. 545-552, 2015.

[15] S. Ray, A. Bhattacharya, and S. Bhattacharjee, "Optimal placement of switches in a radial distribution network for reliability improvement," International Journal of Electrical Power \& Energy Systems, vol. 76, pp. 53-68, 2016

[16] Y. Xu, C.-C. Liu, K. P. Schneider, and D. T. Ton, "Placement of remote-controlled switches to enhance distribution system restoration capability," IEEE Transactions on Power Systems, vol. 31, pp. 11391150, 2016.

[17] I.-h. Lim, T. Sidhu, M. Choi, S. Lee, and B. Ha, "An optimal composition and placement of automatic switches in DAS," IEEE Transactions on Power Delivery, vol. 28, pp. 1474-1482, 2013.

[18] R. Billinton, S. Jonnavithula, "Optimal Switching Device Placement in Radial Distribution Systems", IEEE Transactions on Power Delivery, Vol. 11, pp. 1646-1651, 1996.

[19] Coello Coello, G. Toscano Pulido, and M. Salazar Lechuga, "Handling Multiple Objectives With Particle Swarm Optimization”, IEEE Transactions on Evolutionary Computation, Vol. 8, No. 3, 2004.

[20] El-Fergany and A. Y. Abdelaziz, "Artificial bee colony algorithm to allocate fixed and switched static shunt capacitors in radial distribution networks", Electric Power Components and Systems, Vol. 42, No. 5, 2014. 